\title{
Dosimetric Impact of Bladder Volume Variation in Radiotherapy for Prostate Cancer - A Pilot Study
}

\section{Athena $\mathbf{L i} \mathrm{KL}^{1}$ and Vincent Wing-Cheung $\mathbf{W u}^{2 *}$}

${ }^{1}$ Department of Radiotherapy, Hong Kong Sanatorium and Hospital, Happy Valley, Hong Kong

${ }^{2}$ Department of Health Technology and Informatics, Hong Kong Polytechnic University, Hung Hom, Kowloon, Hong Kong

\begin{abstract}
Background: In external beam radiotherapy of prostate cancer, the position of prostate is often affected by the bladder volume status due to their close anatomical relationship. This study aimed to evaluate the dosimetric impact of bladder volume deviation from the reference planning volume and to establish the acceptable limit of volume deviation in radiotherapy of prostate cancer.

Methods: A total of 43 sets of CBCT images from prostate cancer patients treated by intensity modulated radiotherapy with full bladder were retrospectively recruited. The corresponding planning $C T$ from these patients was retrieved from which the reference plans and 43 CBCT plans were generated respectively. The bladder volume in each plan was measured and the percentage difference of bladder volume between CBCT and planning CT $\left(\% \mathrm{~d}_{\mathrm{BV}}\right)$ was calculated. The CBCT plans were stratified into 12 groups based on the magnitude of $\% \mathrm{~d}_{\mathrm{BV}}$, which ranged from $-75 \%$ to $+75 \%$. In each $\% d_{\mathrm{BV}}$ group, the dose parameters of the CBCT plans were compared with the corresponding reference plans.
\end{abstract}

Results: The $\% \mathrm{~d}_{\mathrm{BV}}$ were ranged from $-79.3 \%$ to $+79.5 \%$. The percentage differences of $\mathrm{D}_{95 \%}$ and $\mathrm{V}_{95 \%}$ of target volume between the CBCT and reference plans decreased significantly with increased $\% \mathrm{~d}_{\mathrm{BV}}$. For the bladder, when the $C B C T$ bladder volume was larger than the reference volume, increase of $\% d_{B V}$ led to slight decrease of $D_{\text {mean }}$, while when the CBCT bladder volume was smaller, the $D_{\text {mean }}$ increased dramatically with decreased $\% d_{B V}$

Conclusion: In radiotherapy of prostate cancer, increase in bladder volume from the reference planning volume led to target underdoes, while decrease in bladder volume increased the bladder dose. Keeping the treatment bladder volume within $\pm 20 \%$ of the reference volume can avoid unacceptable dosimetric outcome.

Keywords: Bladder volume variation; Prostate cancer; Cone beam CT; Radiotherapy; Dosimetric impact

Abbreviations: 2D: 2-Dimensional; 3D: 3-Dimensional; AAA: Analytical Anisotropic Algorithm; CBCT: Cone Beam CT; CT: Computed Tomography; CTV: Clinical Target Volume; D15\%: Dose received by $15 \%$ of the volume; D95\%: Dose received by $95 \%$ of the volume; $\mathrm{D}_{\max }$ : Maximum dose; $\mathrm{D}_{\text {mean }}$ : Mean dose; FOV: Field-of-view; Gy: Gray; HU: Hounsfield Unit; IMRT: Intensity Modulated Radiotherapy; LR: Left-Right; MLC: Multileaf Collimator; MV: Megavoltage; OAR: Organ at Risk; OBI: On-Board Imager; PTV: Planning Target Volume; RTOG: Radiation Therapy Oncology Group; SD: Standard Deviation; SI: Supero-Inferior; V78Gy: Volume of structure receiving 78 Gy

\section{Introduction}

In external beam radiotherapy of prostate cancer, the position of prostate is often affected by the bladder volume status due to the close anatomical relationship of these organs [1]. Interfractional movement and deformation of the prostate resulted from bladder filling were found to induce positional and dosimetric uncertainties of both target volume and organs at risk (OARs) [2,3]. Therefore, a consistent bladder condition during localization and treatment is important. In local department, patients receiving intensity modulated radiotherapy (IMRT) for prostate cancer are usually treated with full bladder and the treatment position is verified by daily orthogonal two-dimensional (2D) imaging and weekly three-dimensional (3D) cone beam computed tomography $(\mathrm{CBCT})$ imaging. In actual treatment, some patients may fail to achieve the required bladder volume due to various reasons including inconsistent residual urine in bladder after voiding, concomitant medications, patient's perception of bladder fullness and patients' state of hydration [4-6]. The situation is more common towards the end of the treatment course when patients have greater difficulty to hold the bladder volume and may intentionally reduce their fluid intake due to increasing radiation induced urinary irritation. As a result, the dose delivered to the target volume and organs at risk may be deviated from the original plan.

Recently, to minimize volume variation, the bladder volume is assessed by a portable ultrasonic scanner before daily positioning. It is a screening procedure before CBCT acquisition to ensure the bladder volume fall within the required limit and avoid repeat of CBCT due to unsatisfactory bladder volume. The assessment allows biofeedback mechanism of daily bladder volume and provides information for drinking advice to the patients [7]. Nevertheless, even so, it is not possible to achieve the exact bladder volume as planned. In local department, patients achieving $\pm 10 \%$ of the planning bladder volume can proceed with the treatment. This is a relatively arbitrary guideline and sometimes not readily achievable by some patients. It will be useful if a threshold bladder volume deviation can be established to guide the treatment delivery. Therefore, this was a preliminary study to evaluate the dosimetric impact of bladder volume deviation on the dosimetric

*Corresponding author: Vincent Wing-Cheung Wu, Department of Health Technology and Informatics, Hong Kong Polytechnic University, Hung Hom, Kowloon, Hong Kong Tel: +85234008567; Fax: +85223624365; E-mail: htvinwu@polyu.edu.hk

Received June 30, 2018; Accepted July 20, 2018; Published July 23, 2018

Citation: Athena Li KL, Wing-Cheung VW (2018) Dosimetric Impact of Bladder Volume Variation in Radiotherapy for Prostate Cancer - A Pilot Study. J Cancer Sci Ther 10: 173-177. doi: 10.4172/1948-5956.1000541

Copyright: (C) 2018 Athena Li KL, et al. This is an open-access article distributed under the terms of the Creative Commons Attribution License, which permits unrestricted use, distribution, and reproduction in any medium, provided the original author and source are credited. 
outcome of the target volume and OARs in IMRT of prostate cancer patients and to establish a threshold deviation value so as to guide the delivery of treatment for the patients.

\section{Methods}

Patients with prostate cancer confined to prostate and seminal vesicle treated by IMRT were retrospectively recruited in this pilot study. These patients were treated with equi-spaced 7 beams (with three beams from anterior/anterior oblique passing through the bladder) using $6 \mathrm{MV}$ photon in a single phase with normal bladder as primary treatment from 2012 to 2014 in the Department of Radiotherapy of Hong Kong Sanatorium and Hospital. The information about target and adjacent organ volumes and doses are shown in Table 1. A total of 43 sets of CBCT images and the corresponding planning CT from the patients were retrieved and studied after obtaining the ethics approval from the hospital and the Hong Kong Polytechnic University.

Planning CT was acquired by a CT simulator (Lightspeed RT 16, GE Medical systems) with slice thickness of $2.5 \mathrm{~mm}$, axial mode, 120 $\mathrm{kV}, 240 \mathrm{~mA}$, data collecting diameter of $50 \mathrm{~cm}$ and $512 \times 512$ matrix covering from mid-abdomen to mid-femurs with patients in supine position. Before CT acquisition, a Foley catheter was inserted into the bladder and about $75-100 \mathrm{~cm}^{3}$ of saline was injected to the bladder to ensure adequate bladder filling. Lopromide (Ultravist 370, Bayer Healthcare) was injected to the balloon of the Foley catheter which helped in the localization of bladder neck and anterior rectal wall. The CT images were sent to the treatment planning system (Eclipse ${ }^{\mathrm{TM}}$, version 10.0, Varian Medical Systems, Palo Alto, CA) where the IMRT plans were computed. The clinical target volume (CTV) including the prostate and seminal vesicle was delineated by the same oncologist for all patients following the same departmental protocol. A margin of $1 \mathrm{~cm}$ was expanded from the CTV in all directions but excluding the rectum forming the planning target volume (PTV) to account for setup uncertainties in treatment delivery. The organs at risk including the bladder, rectum and femoral heads were delineated by the same radiation therapist.

The treatments were delivered by a Truebeam linear accelerator (Varian Medical Systems, Palo Alto, CA) and the treatment position was verified by daily orthogonal $\mathrm{kV}$ imaging and weekly $\mathrm{CBCT}$ acquisition with position correction based on the registration result of planning CT images with the verification images. All the CBCT scans were obtained with half-fan mode, full trajectory from gantry angle of $184.5^{\circ}$ to $175.5^{\circ}, 125 \mathrm{kV}$ and $1056 \mathrm{~mA}$ by the on-board imaging system ( $\mathrm{OBI}^{\circ}$, Varian Medical Systems, Palo Alto, CA). The CBCT images were sent to the treatment planning system where each of them was rigidly registered with the corresponding planning CT in 6 directions using automatic registration algorithm followed by manual adjustment based on bony anatomy and prostate position. All image registration process was performed by a single radiation therapist so as to minimize the inter-operator variation.

\begin{tabular}{|c|c|c|c|c|c|c|}
\hline \multirow{2}{*}{ Variables } & \multicolumn{2}{|c|}{ Planning volume $\left(\mathrm{cm}^{3}\right)$} & \multicolumn{3}{|c|}{ Planned total dose (Gy) } & \multirow{2}{*}{$\begin{array}{l}\text { Number of } \\
\text { fractions }\end{array}$} \\
\hline & Bladder & Rectum & Prostate & sv & PTV & \\
\hline Mean & 327.6 & 125.5 & 75.9 & 66.2 & 73.7 & 42.3 \\
\hline Median & 274.0 & 127.3 & 76.0 & 68.4 & 74.0 & 42.5 \\
\hline SD & 168.5 & 38.3 & 0.8 & 6.0 & 0.8 & 0.8 \\
\hline
\end{tabular}

Table 1: A summary of volume and total planned doses for various structures.
The CTV in the CBCT images, which only included the prostate and seminal vesicles, were also delineated by the same radiation oncologist. Because of the larger field-of-view (FOV) of around $40 \times$ $40 \mathrm{~cm}^{3}$ in $\mathrm{CBCT}$, there were more scatter radiation and artifacts, and the CT numbers for CBCT might be different from conventional CT. Since the relationship between Hounsfield units (HU) of CBCT and electron density for dose calculation was not well established [8] and might lead to error of up to 5\% [9-11], the delivered dose in CBCT was generated using the "Density override technique" and the same CT calibration curve of the CT-simulator in which the density of each structure was assigned according to their corresponding density in planning CT $[9,11]$. For both group of plans, the calculation algorithm was Analytical Anisotropic Algorithm (AAA) and the grid size was 2.5 $\mathrm{mm}^{3}$. According to previous literatures, the dose calculation error of this technique could be kept below $2 \%[12,13]$.

The original IMRT plans of each patient were defined as the reference plans. The IMRT plans were re-calculated using CBCT images with density adjustment by the same planning system as for the original plans with all the planning parameters including the prescribed dose, number of fractions, beam energy, beam configurations, optimization parameters and fluence maps being the same as the original plans. The bladder volume in all the $\mathrm{CBCT}$ and planning $\mathrm{CT}$ images were measured and the percentage difference of bladder volume between CBCT and planning CT, which was represented by $\% \mathrm{~d}_{\mathrm{BV}}$, was calculated. The 43 CBCT plans were stratified into 12 groups based on the magnitude of $\% \mathrm{~d}_{\mathrm{BV}}$, which was ranged from $-75 \%$ to $+75 \%$ with an interval of $15 \%$.

In each $\% \mathrm{~d}_{\mathrm{BV}}$ group, dose parameters for the target volume and bladder were compared between the CBCT plans and the corresponding reference plans in terms of their average percentage differences $(\% \mathrm{~d})$. These dose parameters included $\mathrm{D}_{95 \%}$ (dose received by $95 \%$ volume), $\mathrm{D}_{\max }$ (maximum dose), $\mathrm{V}_{95 \%}$ (volume receiving $95 \%$ of prescribed dose) and $\mathrm{D}_{\text {mean }}$ (mean dose) of the target volume; $\mathrm{D}_{\max }$, $\mathrm{D}_{\text {mean, }}, \mathrm{D}_{15 \%}, \mathrm{D}_{25 \%}, \mathrm{D}_{35 \%}, \mathrm{D}_{50 \%}$ (dose received by $15 \%, 25 \%, 35 \%$ and $50 \%$ respectively) and $\mathrm{V}_{78 \mathrm{~Gy}}$ (volume receiving $78 \mathrm{~Gy}$ ) of the bladder. $\mathrm{V}_{78 \mathrm{~Gy}}$ was chosen as one of the assessment parameters because it was reported that 78 Gy was regarded as the dose tolerance for bladder tissue, exceeding this limit would increase the risk of both early and late urinary toxicities [14-16]. CBCT plans with $\mathrm{D}_{95 \%}$ of target volume lower than $90 \%$ of its value in the reference plans were considered as "underdose". Also, with reference to the Radiation Therapy Oncology Group (RTOG) guidelines, bladder dose that fell under either one of the following conditions: $\mathrm{D}_{\text {mean }}$ increase of over $25 \%$, increase of $\mathrm{D}_{15 \%}$, $\mathrm{D}_{25 \%}, \mathrm{D}_{35 \%}$ or $\mathrm{D}_{50 \%}$ of over $50 \%$ were regarded as "overdose". The level of $\% \mathrm{~d}_{\mathrm{BV}}$ that started to show target underdose and bladder overdose was identified as the threshold bladder volume deviation. All statistical tests were performed using GraphPad Software (PRISM ${ }^{\oplus}$, Version 6.0c). D'Agostino-Pearson normality tests were carried out for all the parameters to check for normal distribution. Pearson correlation tests or Spearman correlation tests were performed to study the correlation between the dose parameters and the average $\% \mathrm{~d}_{\mathrm{BV}}$ in each group.

\section{Results}

The result of D'Agostino-Pearson normality test showed that the distribution of $\% \mathrm{~d}_{\mathrm{BV}}$ followed Gaussian distribution $(\mathrm{p}=0.231)$. The $\% \mathrm{~d}_{\mathrm{BV}}$ was ranged from $-79.3 \%$ to $+79.5 \%$ with the mean, median and standard deviation (SD) of $-6.5 \%,-13.5 \%$ and $41.0 \%$ respectively. Twenty-six out of the 43 cases (60.5\%) presented with a smaller bladder volume in the CBCT plan relative to the planning CT plans (i.e., negative $\% \mathrm{~d}_{\mathrm{BV}}$ value). The stratification of CBCT plans are shown in Table 2. 


\begin{tabular}{|c|c|c|}
\hline Group & Average \% $_{\mathbf{B v}}$ & Number of Subjects \\
\hline$<-75 \%$ & -79.3 & 1 \\
\hline$-75 \%$ to $-60 \%$ & -66.0 & 3 \\
\hline$-60 \%$ to $-45 \%$ & -50.3 & 4 \\
\hline$-45 \%$ to $-30 \%$ & -35.8 & 7 \\
\hline$-30 \%$ to $-15 \%$ & -22.6 & 5 \\
\hline$-15 \%$ to $0 \%$ & -8.7 & 6 \\
\hline $0 \%$ to $15 \%$ & 7.1 & 4 \\
\hline $15 \%$ to $30 \%$ & 18.4 & 4 \\
\hline $30 \%$ to $45 \%$ & 38.1 & 4 \\
\hline $45 \%$ to $60 \%$ & 54.9 & 1 \\
\hline $60 \%$ to $75 \%$ & 67.3 & 1 \\
\hline$>75 \%$ & 79.5 & \\
\hline
\end{tabular}

Table 2: Stratification of the CBCT plans based on magnitude of percentage bladder volume variations.

\begin{tabular}{|c|c|c|c|c|}
\hline \multicolumn{2}{|c|}{ Dose parameters } & $\mathbf{r}$ & Spearman $\mathbf{r}$ & ${ }^{*} \mathbf{p}$ \\
\hline \multirow{4}{*}{ Target } & $\mathrm{V}_{95 \%}$ & -0.889 & $\mathrm{~N} / \mathrm{A}$ & $<0.001$ \\
\cline { 2 - 5 } & $\mathrm{D}_{95 \%}$ & -0.878 & $\mathrm{~N} / \mathrm{A}$ & $<0.001$ \\
\cline { 2 - 5 } & $\mathrm{D}_{\text {mean }}$ & -0.842 & $\mathrm{~N} / \mathrm{A}$ & $<0.001$ \\
\cline { 2 - 5 } & $\mathrm{D}_{\max }$ & -0.771 & $\mathrm{~N} / \mathrm{A}$ & 0.003 \\
\hline \multirow{4}{*}{ Bladder } & $\mathrm{D}_{\max }$ & -0.563 & $\mathrm{~N} / \mathrm{A}$ & 0.057 \\
\cline { 2 - 5 } & $\mathrm{D}_{\text {mean }}$ & -0.907 & $\mathrm{~N} / \mathrm{A}$ & $<0.001$ \\
\cline { 2 - 5 } & $\mathrm{D}_{15 \%}$ & -0.862 & $\mathrm{~N} / \mathrm{A}$ & $<0.001$ \\
\cline { 2 - 5 } & $\mathrm{D}_{25 \%}$ & -0.881 & $\mathrm{~N} / \mathrm{A}$ & $<0.001$ \\
\cline { 2 - 5 } & $\mathrm{D}_{35 \%}$ & $\mathrm{~N} / \mathrm{A}$ & -0.965 & $<0.001$ \\
\cline { 2 - 5 } & $\mathrm{D}_{50 \%}$ & -0.845 & $\mathrm{~N} / \mathrm{A}$ & 0.001 \\
\cline { 2 - 5 } & $\mathrm{V}_{786 \mathrm{y}}$ & $\mathrm{N} / \mathrm{A}$ & -0.546 & 0.071 \\
\hline \multirow{yyyy}{*}{} & & & & \\
\hline
\end{tabular}

*The correlation with $p$-value $<0.05$ was considered statistically significant

Table 3: Results of correlation tests between $\% \mathrm{~d}_{\mathrm{BV}}$ and dose parameters.

\section{Target volume dose}

$\% \mathrm{~d}$ of $\mathrm{D}_{95 \%}$ and $\mathrm{V}_{95 \%}$ of target volume between in the CBCT were smaller than the reference plans. They decreased significantly from $\% \mathrm{~d}_{\mathrm{BV}}=-75 \%$ with increasing $\% \mathrm{~d}_{\mathrm{BV}}$ (Figure 1 ) and there were large fluctuations when the $\% \mathrm{~d}_{\mathrm{BV}}$ ranged from $20 \%$ to $80 \%$. The $\% \mathrm{~d}$ of $\mathrm{D}_{\text {mean }}$ also decreased with increasing $\% \mathrm{~d}_{\mathrm{BV}}$ but with smaller magnitude and fluctuations, while the $\% \mathrm{~d}$ of $\mathrm{D}_{\max }$ did not vary much with $\% \mathrm{~d}_{\mathrm{BV}}$. The results of the correlation tests showed that there were significant correlations between the average $\% \mathrm{~d}_{\mathrm{BV}}$ of each group and all the target dose parameters (Table 3). In general, $60.5 \%$ of CBCT plans had their $\mathrm{D}_{95 \%}$ lower than that of the reference plans. CBCT plans with bladder volume larger than the reference plans were more susceptible to target volume underdose $(61.5 \%)$, in which $90.8 \%$ of the under-dose was more than $10 \%$.

\section{Bladder dose}

The average $\% \mathrm{~d}$ of $\mathrm{D}_{\max }$ and $\mathrm{D}_{\text {mean }}$ of bladder in each group ranged from $-0.6 \%$ to $1.6 \%$ and $-24.7 \%$ to $162 \%$ respectively (Figure 2 ). In the region of positive $\% \mathrm{~d}_{\mathrm{BV}}$ (bladder volume of CBCT plan>reference plan), greater $\% \mathrm{~d}_{\mathrm{BV}}$ led to slight decrease of $\mathrm{D}_{\text {mean }}$, while in the region of negative $\% \mathrm{~d}_{\mathrm{BV}}$, the $\mathrm{D}_{\text {mean }}$ increased dramatically with decreasing $\% \mathrm{~d}_{\mathrm{BV}}$. For the most extreme case, there was $162 \%$ increase in the $\mathrm{D}_{\text {mean }}$ when $\% \mathrm{~d}_{\mathrm{BV}}$ was $-79.3 \%$. Results of Pearson correlation tests suggested that there was strong correlation between the $\% \mathrm{~d}_{B V}$ and $\% \mathrm{~d}$ of $\mathrm{D}_{\text {mean }}(\mathrm{r}=-$ 0.907 and $\mathrm{p}<0.001$ ) but no correlation between the $\% \mathrm{~d}_{\mathrm{BV}}$ and $\% \mathrm{~d}$ of $\mathrm{D}_{\max }$ (Table 3). The average $\% \mathrm{~d}$ of $\mathrm{D}_{15 \%}, \mathrm{D}_{25 \%}, \mathrm{D}_{35 \%}$ and $\mathrm{D}_{50 \%}$ did not vary much with positive $\% \mathrm{~d}_{\mathrm{BV}}$ but increased dramatically with decreasing $\% \mathrm{~d}_{\mathrm{BV}}$ when $\% \mathrm{~d}_{\mathrm{BV}}$ was negative (Figure 3 ). Significant correlations

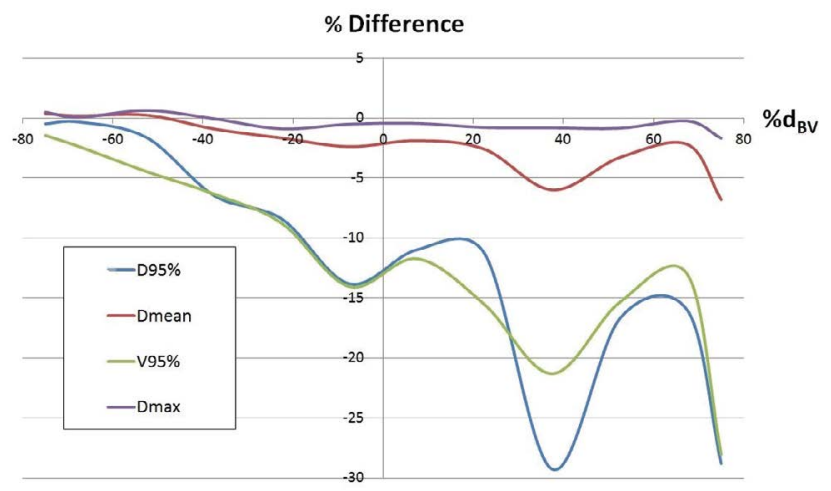

Figure 1: The percentage changes (y-axis) of target dose parameters with respect to the average percentage change of bladder volume between treatment and planning $\left(\% \mathrm{~d}_{\mathrm{BV}}\right)$.

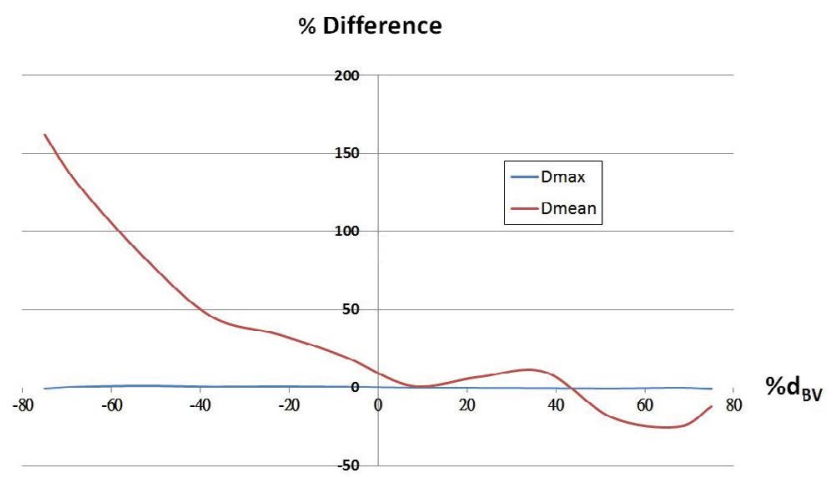

Figure 2: The percentage changes ( $y$-axis) of bladder dose parameters $\left(D_{\text {max }}\right.$ and $D_{\text {mean }}$ ) with respect to the average percentage change of bladder volume between treatment and planning $\left(\% \mathrm{~d}_{\mathrm{BV}}\right)$.

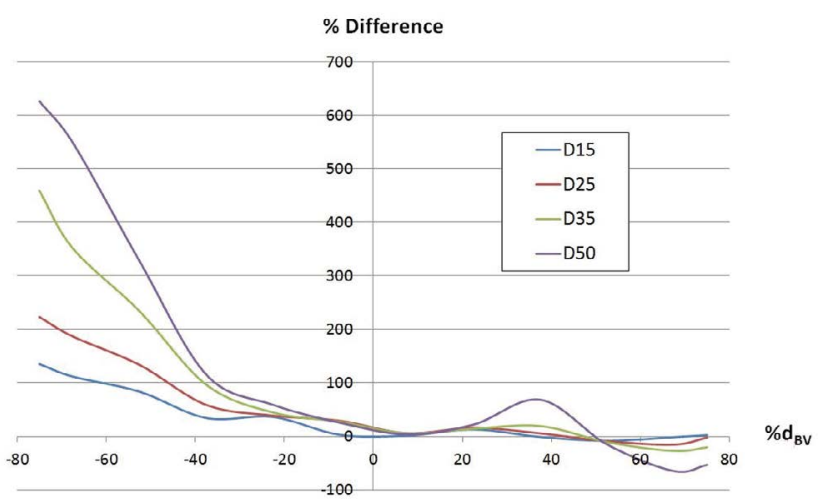

Figure 3: The percentage changes (y-axis) of bladder dose parameters $\left(D_{15}\right.$ $-D_{50}$ ) with respect to the average percentage change of bladder volume between treatment and planning $\left(\% \mathrm{~d}_{\mathrm{BV}}\right)$.

between $\% d$ of $D_{15 \%}, D_{25 \%}, D_{35 \%}$ and $D_{50 \%}$ with the average $\% d_{B V}$ in each group were also found $(\mathrm{r}<-0.8$ and $\mathrm{p}<0.001)$ (Table 3$)$. For $\mathrm{V}_{78 \mathrm{~Gy}}$, it did not change with $\% \mathrm{~d}_{\mathrm{BV}}$ obviously when the $\% \mathrm{~d}_{\mathrm{BV}}$ was positive but fluctuated considerably when it became negative, with a peak when $\% \mathrm{~d}_{\mathrm{BV}}$ was around $-50 \%$ (Figure 4 ). No significant correlation between $\mathrm{V}_{78 \mathrm{~Gy}}$ and $\% \mathrm{~d}_{\mathrm{BV}}$ was found $(\mathrm{p}=0.071)$. 


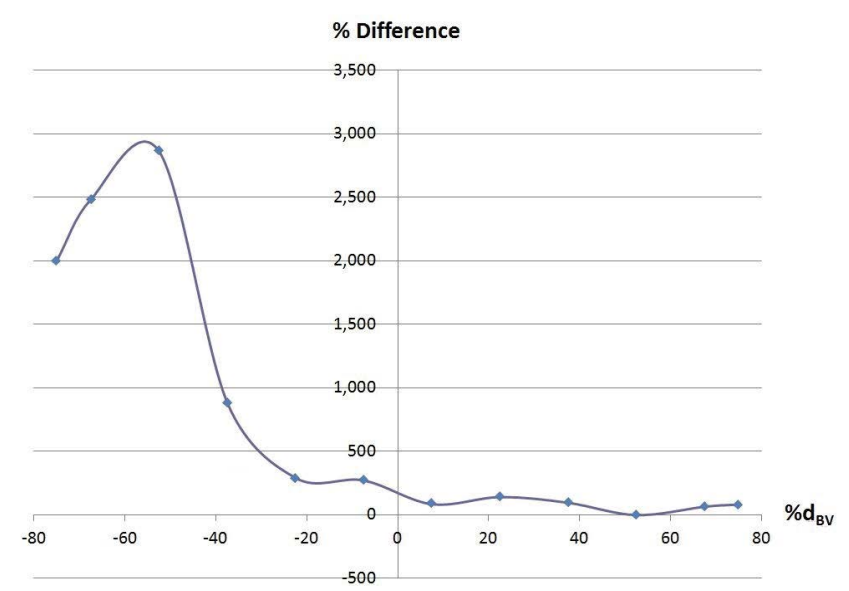

Figure 4: The percentage changes ( $y$-axis) of $V_{786 y}$ of the bladder with respect to the average percentage change of bladder volume between treatment and planning $\left(\% \mathrm{~d}_{\mathrm{BV}}\right)$.

\section{Discussion}

\section{Target dose}

The target doses such as $\mathrm{D}_{95 \%}, \mathrm{~V}_{95 \%}$ and $\mathrm{D}_{\text {mean }}$ significantly decreased with increasing $\% \mathrm{~d}_{\mathrm{BV}}$. It was observed in our study and reported by some studies that the prostate was displaced anteriorly with decreased bladder volume and posteriorly with increased bladder volume $[17,18]$. With the change of bladder volume during the treatment course, the overlap regions between the PTV and bladder varied, and these were observed between the CBCT image and the reference CT image, which contributed to the deviation of dose distribution to the target volume. When the CBCT bladder volume was larger than that of reference plan, the target dose continuously decreased with increasing $\% \mathrm{~d}_{\text {BV }}$ because the prostate was further displaced posteriorly by the more distended bladder and induced posterior displacement of prostate. As a result, with increasing $\% \mathrm{~d}_{\mathrm{BV}}$, the prostate was pushed away from the prescribed high dose volume and led to a decreased target dose. However, $\mathrm{D}_{\max }$ of target did not vary much with $\% \mathrm{~d}_{\mathrm{BV}}$ because it was the hot spot of the target which still existed even when a portion of the target was pushed away from the original high dose volume. The impacts on $\mathrm{D}_{95 \%}$ and $\mathrm{V}_{95 \%}$ with $\% \mathrm{~d}_{\mathrm{BV}}$ were more obvious than $\mathrm{D}_{\text {mean }}$, because they were more sensitive to the presence of target underdoes induced by the positional displacement. On the other hand, since $\mathrm{D}_{\text {mean }}$ was the average dose of the whole target, it was relatively less affected by under-does volume.

When the bladder volume was smaller than the reference volume (i.e., negative $\% \mathrm{~d}_{\mathrm{BV}}$ ), the impact on the target dose was less significant. With a smaller bladder, the prostate mainly displaced anteriorly following the anterior displacement of posterior bladder wall. At the same time, the displacements of prostate in the supero-inferior (SI) and left-right (LR) directions were negligible $[17,19]$. On the other hand, greater fluctuations in $\% \mathrm{~d}$ of $\mathrm{D}_{95 \%}$ and $\mathrm{V}_{95 \%}$ were observed at the positive side of the curves. It was because when the bladder became bigger, it would force the prostate to display posteriorly and inferiorly in a much greater extent than those caused by the shrinkage of the bladder. As a result, the geometric deviation of the target could vary considerably from the reference position leading to much greater impact on the target dose with larger fluctuations.

A limitation of this study was that the impact of the rectal volume change was not included, as the target volume position might also be affected by the rectal condition, which partly accounted for the fluctuations of the target doses. However, according to the recent study from Chen et al. [20], the rectal volume variations were much less obvious than those of the bladder, it was expected that the dosimetric impact from the rectum status would be relatively small.

\section{Bladder dose}

Over $60 \%$ of the CBCT images showed a reduced bladder volume in our study. The reason for higher occurrence of a reduced bladder could be because of the radiation induced urinary toxicities including irritation of urinary epithelium, increasing urgency of urination and decreased ability in maintaining the required bladder filling. In this study, the bladder dose increased significantly with reduction in bladder volume compared with the reference volume (i.e., negative $\% \mathrm{~d}_{\mathrm{BV}}$ ), which was in line with a few previous studies $[4,5,17,21]$. A shrunken bladder would lead to a greater percentage volume covered by the high dose region, resulting in higher mean dose. The increase magnitude of $\mathrm{D}_{\text {mean }}$ was up to nearly two folds when the reduction of volume was up to $75 \%$. Furthermore, the increase of $\mathrm{V}_{78 \mathrm{~Gy}}$ of the bladder due to bladder volume reduction could be up to nearly 30 times indicating a much higher risk of radiation induced urinary toxicities when the bladder volume was smaller than expected. On the other hand, expansion of the bladder from the original reference volume did not lead to significant change of bladder dose. It was because patients in this cohort were already treated with full bladder; further increase in bladder volume would not greatly change the percentage volume of bladder that already received high dose. Besides, the reason for $\mathrm{D}_{\max }$ that remained unchanged in all $\% \mathrm{~d}_{\mathrm{BV}}$ was because the maximum dose was always present at the point where the bladder was in close proximity with the target regardless of the bladder volume. Overall, our results indicated that shrinkage of the bladder during treatment would bring more detrimental effects to the patients than those with bladder expansion due to the much-increased bladder dose.

With regard to the target dose, any bladder volume deviation during treatment had led to a reduction of target dose. According to our set criteria of target underdoes, in which a $10 \%$ reduction in $\mathrm{D}_{95 \%}$ and $\mathrm{D}_{\text {mean }}$ of the target was regarded as the tolerance, a bladder volume increases of over $20 \%$ from the reference plan would not be acceptable (Figure 1). On the other hand, decrease of bladder volume during treatment caused overdose of the bladder. Based on the earlier stated guidelines, bladder volume reduction of over $20 \%$ from the reference plan would not be accepted. In other word, we recommend that in the radiotherapy of prostate cancer patients, it is advisable to keep the treatment bladder volume within $\pm 20 \%$ of the reference volume. In addition, although it has been accepted that treatment with full bladder could spare the bladder and bowel, excessive full bladder during planning should not be encouraged because it might be difficult for patients to maintain such a condition and failure to achieve that bladder volume would lead to unwanted bladder complications. Actually, it was reported that bladder volume larger than $150 \mathrm{~cm}^{3}$ did not further enhance the sparing of organs at risk. Therefore, an optimal planning bladder volume is one which can satisfy the dose constraints for the organs at risk, and also maximize the compliance of the required bladder volume by the patients during treatment so that the dosimetric outcome will not be compromised. Furthermore, this study can be extended to the radiotherapy of other pelvic tumours such as cervical and rectal cancers so as to establish a threshold bladder volume variation percentage during treatment. 


\section{Conclusion}

In radiotherapy of prostate cancer, increase in treatment bladder volume from the reference planning volume increased the chance of target underdoes while decrease in treatment bladder volume increased the bladder dose with increased risk of urinary toxicities. Since the influence of bladder volume deviation on the target and bladder doses was more significant when the deviation exceeded $\pm 20 \%$ of the reference volume, we recommend keeping the treatment bladder volume within this limit in IMRT of prostate cancer.

\section{Declarations}

\section{Ethics approval}

Ethics approval from the Hong Kong Sanatorium and Hospital and the Hong Kong Polytechnic University (Reference No: HSEARS20150626001).

\section{Consent for publication}

Not applicable.

\section{Availability of data and materials}

Materials in the manuscript are available by contacting the author at htvinuw@polyu.edu.hk.

\section{Competing interests}

There is no financial or non-financial competing interest for all authors.

\section{Funding}

Nil.

\section{Authors' contributions}

AKL Li: Design of study, retrieval of subject data, collection and analysis of data, draft of manuscript; Vincent WC Wu: Design of study, data analysis, final editing and paper submission.

\section{Acknowledgements}

Nil.

\section{References}

1. Nakamura N, Shikama N, Takahashi O, Ito M, Hashimoto M, et al. (2010) Variability in bladder volumes of full bladders in definitive radiotherapy for cases of localized prostate cancer. Strahlenther Onkol 186: 637-642.

2. Nakamura N, Shikama N, Takahashi O, Sekiguchi K, Hama Y, et al. (2012) The relationship between the bladder volume and optimal treatment planning in definitive radiotherapy for localized prostate cancer. Acta Oncol 51: 730-734

3. Bell LJ, Cox J, Eade T, Rinks M, Kneebone A (2014) The impact of rectal and bladder variability on target coverage during post-prostatectomy intensity modulated radiotherapy. Radiother Oncol 110: 245-250.

4. Moiseenko V, Liu M, Kristensen S, Gelowitz G, Berthelet E (2007) Effect of bladder filling on doses to prostate and organs at risk: A treatment planning study. J Appl Clin Med Phys 8: 55-68.
5. O'Doherty UM, Mcnair HA, Norman AR, Miles E, Hooper S, et al. (2015) Variability of bladder filling in patients receiving radical radiotherapy to the prostate. Radiother Oncol 79: 335-340.

6. Hynds S, Mcgarry CK, Mitchell DM, Early S, Shum L, et al. (2011) Assessing the daily consistency of bladder filling using an ultrasonic bladder scan device in men receiving radical conformal radiotherapy for prostate cancer. $\mathrm{Br} \mathrm{J}$ Radio 84: 813-818.

7. Stam MR, Thvan Lin EN, Van der Vight LP, Kaanders JH, Visser AG (2006) Bladder filling variation during radiation treatment of prostate cancer: Can the use of a bladder ultrasound scanner and biofeedback optimize bladder filling? Int J Radiat Oncol Biol Phys 65: 371-377.

8. Yoo S, Yin F (2006) Dosimetric feasibility of cone-beam CT-based treatment planning compared to CT-based treatment planning. Int J Radiat Oncol Biol Phys 66: 1553-1561.

9. Fotina I, Hopfgartner J, Stock M, Steininger T, Lütgendorf-caucig C, et al (2012) Feasibility of CBCT-based dose calculation: Comparative analysis of HU adjustment techniques. Radiother Oncol 104: 249-256.

10. Moteabbed M, Sharp GC, Wang Y, Trofimov A, Efstathiou JA, et al. (2015) Validation of a deformable image registration technique for cone beam CTbased dose verification. Med Phys 42: 196-205.

11. Van Zijtveld M, Dirkx M, Heijmen B (2007) Correction of conebeam CT values using a planning CT for derivation of the "dose of the day". Radiother Oncol 85: $195-200$.

12. Doemer A, Chetty IJ, Glide-hurst C, Nurushev T, Hearshen D, et al. (2015) Evaluating organ delineation, dose calculation and daily localization in an openMRI simulation workflow for prostate cancer patients. Radiat Oncol 10: 37

13. Lee YK, Bollet M, Charles-Edwards G, Flower MA, Leach MO, et al. (2003) Radiotherapy treatment planning of prostate cancer using magnetic resonance imaging alone. Radiother Oncol 66: 203-216.

14. Heemsbergen WD, Al-Mamgani A, Witte MG, Van Herk M, Pos FJ, et al. (2010) Urinary obstruction in prostate cancer patients from the dutch trial 68 Gy vs. 78 Gy): Relationships with local dose, acute effects, and baseline characteristics. Int J Radiat Oncol Biol Phys 78: 19-25.

15. Peeters ST, Heemsbergen WD, Van Putten WL, Slot A, Tabak H, et al. (2005) Acute and late complications after radiotherapy for prostate cancer: Results of a multicenter randomized trial comparing 68 Gy to 78 Gy. Int J Radiat Oncol Biol Phys 61: 1019-1034.

16. Cheung M, Tucker S, Dong L, De Crevoisier R, Lee AK, et al. (2007) Investigation of bladder dose and volume factors influencing late urinary toxicity after external beam radiotherapy for prostate cancer. Int J Radiat Oncol Biol Phys 67: 1059-1065.

17. Cambria R, Jereczek-fossa B, Zerini D, Cattani F, Serafini F, et al. (2011) Physical and clinical implications of radiotherapy treatment of prostate cancer using a full bladder protocol. Strahlenther Onkol 187: 799-805.

18. Deurloo KE, Steenbakkers RJ, Zijp LJ, De Bois JA, Nowak PJ, et al. (2005) Quantification of shape variation of prostate and seminal vesicles during external beam radiotherapy. Int J Radiat Oncol Biol Phys 61: 228-238.

19. Pinkawa M, Asadpour B, Siluschek J, Gagel B, Piroth MD, et al. (2007) Bladde extension variability during pelvic external beam radiotherapy with a full or empty bladder. Radiother Oncol 83: 163-167.

20. Chen Z, Yang Z, Wang J, Hu W (2016) Dosimetric impact of different bladder and rectum filling during prostate cancer radiotherapy. Radiat Oncol 11: 103

21. Zellars RC, Roberson PL, Strawderman M, Zhang D, Sandler HM, et al. (2000) Prostate position late in the course of external beam therapy: Patterns and predictors. Int J Radiat Oncol Biol Phys 47: 655-660. 